\title{
Physical Significance of Measure
}

\author{
Jody A. Geiger \\ Informativity Institute, Chicago, IL, USA \\ Email: jodygeiger@informativity.org
}

How to cite this paper: Geiger, J.A. (2020) Physical Significance of Measure. Journal of High Energy Physics, Gravitation and Cosmology, 6, 62-89.

https://doi.org/10.4236/jhepgc. 2020.61008

Received: December 9, 2019

Accepted: January 12, 2020

Published: January 15, 2020

Copyright (c) 2020 by author(s) and Scientific Research Publishing Inc. This work is licensed under the Creative Commons Attribution International License (CC BY 4.0).

http://creativecommons.org/licenses/by/4.0/

\begin{abstract}
Providing for a comprehensive model of physics that describes both the discrete and non-discrete behavior of matter has proved difficult and elusive. Using a new approach, we express Heisenberg's uncertainty principle in terms of measure and counts of those measures to resolve an expression consisting entirely of counts. Three arguments are presented each identifying one property of measure. Firstly, the three measures-length, mass and time-are each shown to have a physically significant lower bound. Secondly, each measure is shown to be discrete throughout the entire measurement domain. And thirdly, measure is shown to be the result of three frames of reference: the observer, the observed and the universe. Using these observations alone, the model resolves values for Planck's constant, the gravitational constant and gravitational curvature.
\end{abstract}

\section{Keywords}

Measure, Measurement Quantization, Gravity, Planck Units, Discrete, Discrete Measure

\section{Introduction}

A bounded system with discrete behavior may be identified anywhere there is a count of a reference behavior. Notably, understanding discreteness requires that we identify the reference and that will require a nomenclature that accommodates both discrete and non-discrete terms. We begin by introducing a second set of terms alongside the first, the two terms each capable of following a distinct set of mathematical operators.

The proposed approach achieves the design such that there are two terms for each measure, the reference measure and some count of that reference. Initially, Planck's Units are used as a guide in understanding the reference measures, length, mass and time. For the second term, the letter $n$ followed by the corres- 
ponding subscript for each measure, $n_{L}, n_{M}$ and $n_{T}$ is used to describe counts of the reference measures. Combined, the new nomenclature allows us to distinguish discrete from non-discrete properties of a phenomenon without assuming the composition. That is to emphasize, the values of the measures do not need to be resolved. Nor is it necessary to assume that the measures are the smallest physically significant measures or that the counts are integer. The operators, values and discreteness of each term are resolved entirely from the physical record.

Of notable interest are commonly used physical expressions that may be described without measure. That is, we may reduce expressions, such as escape velocity and the Heisenberg uncertainty principle, using equivalent representations of reference measures and counts of those measures such that only the count terms remain. The reference measures cancel out leaving commensurable dimensionless relations, an interesting result that quickly distinguishes dimensional expressions from geometric phenomena. For this reason, dimensional homogeneity requires a broader understanding than the usual constraints encountered when working only with measure.

To correlate this new approach to a specific phenomenon, we focus our attention on Heisenberg's uncertainty principle. Reducing the expression allows us to present a three-tiered argument. That is, that each measure has a physically significant lower bound, that measure is discrete throughout the entire physical domain and that measure is a function of three frames of reference.

With respect to frames of reference, measure has been portrayed historically as a function of the observer and the observed with a description of light anchoring a third frame, which we will call the universe for lack of a more physically specific term. This traditional approach is not ideal as it constrains our understanding of measure to just one aspect of the universal frame, a description of length with respect to time $c=1 / t$. Of equal importance are the boundary relations $m / t$ and $1 / m$. When incorporating all three terms with respect to the three frames of reference, an expanded understanding of the relationship between measure and that which is measured is achieved.

In Section III we apply the principles of discrete measure to the description of phenomena. Several predictions are resolved such as a quantum description of gravity, a new approach to resolving the gravitational constant $G$, Planck's reduced constant $\hbar$, specific polarization measures necessary for the quantum entanglement of X-rays and the previously discussed quantum presentation of Heisenberg's uncertainty principle.

Also addressed are the obstacles met by Planck and others in the search for a physically significant connection to Planck's Units. This requires a gravitationally independent approach to resolving Planck's constant. And with that, both discrete and non-discrete solutions to $\hbar$ can be used to resolve $G$.

The results are as expected. Only the discrete approach predicts a value for $G$ accurate to six significant digits. The non-discrete approach is measurably in- 
correct. Furthermore, support for discrete measure resolves one more hurdle. It constrains measure to whole-unit counts of the reference measures, eliminating singularities.

Further reading and explanation of each of these phenomena are available in the published record [1] [2] [3]. The focus of this paper is not with respect to applications of measurement quantization, but to discuss the foundations of modern theory, what is measure and how do bounds to measure lead to discrete behavior.

\section{Methods}

\subsection{Theoretical Landscape}

A review of the theoretical landscape with respect to a discussion of the foundations of modern theory is difficult. But that is not to say that physicists are not fully engaged in questioning the nature of spacetime, the qualities and/or properties of space and time or their emergence. For this, there is a great deal of literature. Thus, such literature does have a significant presence. Although answers to some of these inquiries are addressed, the questions we begin with are more basic. We might say the question we begin with is often singular. For instance, what is measure?

It is in this more constrained light that the theoretical landscape is sparse. Yes, modern theory does address the relative nature of measure, that the dimensions length, mass and time are correlated, but to date there is no expression describing their collective correlation without the introduction of several constants. This is quite surprising given the limited number of solutions to their relation. For instance, wouldn't one just introduce a constant $x$ and seek to understand its physical significance: $l m=x t$ ?

Perhaps. But, as simple as this may be, this has not stopped modern theorists from developing more complex models, some of which have been immensely successful. Namely, the most notable of these is relativity or of particular interest Special Relativity (SR) which describes the relation between an observer and a target as geometry. Typically, the observer sits in a conceptual framework referred to as the inertial frame of reference, or more specifically a state not experiencing the action of force. The model is very accurate. But, is it the most correct model considering several issues regarding the appearance of singularities?

This again depends on the questions we are asking. Consider yet a new line of inquiry. At the base of SR, we have mathematical principles which depend on and originate around use of the Pythagorean Theorem. We have a concept of frames of reference and some prerequisite properties which we have assigned or at least with which we identify these frames of reference. But, how many frames of reference should there be? Exactly why are there one, two or even more frames of reference? One might conjecture, for instance that the number of frames important to the concept of measure should, after all, be a function of the Pythagorean Theorem, which has three terms. Thus, are there not three frames 
of reference and if this is readily agreed to, then how have we evolved modern theory to account for the behavior of matter with respect to each of the three frames?

We offer these thoughts only to entice the reader to consider that measure is not well understood. And while definitive answers can be provided, the greater goal is also to bring to the reader's attention that it is very difficult to provide a literary context for a subject that has not been addressed. Specifically, modern theorists by-in-large focus on the physical observations of virtual particles in a vacuum, on the discrete behavior of phenomena in nature, on symmetries, and finally in investigations that encompass an expansive field which we may collectively aggregate under the title of mathematical fitting of measurable free variables. Examples of this latter field include modified gravity and Modified Newtonian Dynamics (MOND) but extend also to include approaches that adapt complex mathematical descriptions to observational data; for instance, String Theory, Loop Quantum Gravity (LQG), Supersymmetry (SUSY) or even something as tangible as $\Lambda \mathrm{CDM}$. Are these approaches any less important than the old school classical approaches that gave rise to SR?

Before proceeding, it would be most appropriate to call out LQG as a formidable model of physical theory built on the idea that nature is discrete. With respect to the research presented within, this approach is in part accurate and askew in only one minor, but very important way. The mathematical tools used in LQG also become less useful in consideration of this research where only the simplest concepts from classical mechanics suffice. That said, LQG is a pioneering field that has offered many insights into the behavior of phenomena and should not be underestimated. The reasons behind this will become clearer as we proceed.

With respect to the theoretical landscape, we may agree that there is legitimate and significant work occurring in all of these approaches. But we add that meaning, that thing which provides a complete picture, a comprehensive explanation of physical phenomena that is universal across all fields of modern theory cannot arise from the fitting of free variables to a generalized model describing what is observed. Rather, it is the role of theory to identify inarguable principles that reside below the data that give rise to the laws which we use to describe the behavior of matter.

To demonstrate the importance of this need, consider the case of a physicist that delivers an expression ([3], Eq. 66) describing the orbital dynamics of stars about a galactic core. Would we not still ask why this expression exists? Or, at least, should we not ask what its relation is to the other laws which describe planetary orbital dynamics, localized gravity or at least apples falling from trees? This is the role of theory, and so once again we find ourselves asking, what is the theoretical landscape of the published literature that addresses the fundamental questions underneath the fundamental questions?

And while we may argue that there exists an entire field of study called measurement theory and even yet the very successful field of LQG, it is with this 
grander view of physical endeavor that we bring into focus a new question which reminds the reader how a model should work. Why are there 26 constants?

And thus, we must start over yet again, but this time we begin with nothing. To be more precise, we assume nothing about measure with a nomenclature that allows for a variety of possibilities. In this approach, we introduce the idea of a fundamental measure and counts of those measures. For example, time would then be described as $t=n_{T} t_{f}$ such that $n_{T}$ is some count of a fundamental unit of time $t_{f}$ Bear in mind, we assume nothing about the values of either the counts or the measures. Both may vary, take on any value, be found entirely redundant or physically meaningless.

With this new nomenclature, we then present descriptions of phenomena. Carefully selected, we resolve the count and measure values. More importantly, we resolve why the values exist and where they are applicable. This is the approach taken in this paper and it would be inappropriate to address those findings without carefully leading the reader through the physical support on which our conclusions are based.

That said, there exists one exception important to this discussion. The approach demonstrates that objects in the universe behave non-discretely, but there are bounds to measure that constrain the precision of those observations. Moreover, the behavior of matter follows not the non-discrete frame, but the discrete frame of the third frame. This is a shared property of the observer's frame best described by Heisenberg's Uncertainty Principle. What is less obvious is that a mathematical description of nature that is discrete and a mathematical description of nature that is non-discrete can be combined in such a way to realize their difference. It is this difference that defines and allows us to resolve the constants of nature (the upper and lower bound relations between the counts of the fundamental measures) and the laws of nature (more complex relations that may be reduced to an expression describing the relation of the fundamental measures). And it is as such that we are no longer approaching modern theory with expressions that describe nature, but a wholistic approach that can resolve the constants and expressions themselves from a more fundamental model of understanding. For this, there is no theoretical landscape.

\subsection{Physical Significance of Measure}

In modern theory, the expressions we use to describe the behavior of matter are presumed to be physically significant to any mathematical precision. But, we also recognize that measure has a lower bound, a measure where the effects described by quantum mechanics constrain the precision of measure with respect to the length, mass and elapsed time associated with a phenomenon. Heisenberg's Uncertainty Principle is one tool we may use to identify the scope of a physically significant measurement domain. Before we begin, we recognize that light may be described as a count $n_{L}$ of length units $l_{p}$ divided by a count $n_{T}$ of time units $t_{p}, c=n_{L} l_{p} / n_{T} t_{p}$ such that 


$$
n_{L}=n_{T} .
$$

We also recognize Planck's unit expressions for length $l_{p}$ and mass $m_{p}$ which serve as reasonably accurate dimensional approximations describing measure.

$$
\begin{aligned}
& l_{p}=\left(\frac{\hbar G}{c^{3}}\right)^{1 / 2}, \\
& m_{p}=\left(\frac{\hbar c}{G}\right)^{1 / 2} .
\end{aligned}
$$

For $c=l_{p} / t_{p}$ and the above two Planck expressions, we resolve that their product is

$$
\begin{gathered}
l_{p}^{2} m_{p}^{2}=\frac{\hbar c}{G} \frac{\hbar G}{c^{3}}=\frac{\hbar^{2}}{c^{2}}, \\
\hbar=c l_{p} m_{p}=\frac{l_{p}^{2} m_{p}}{t_{p}} .
\end{gathered}
$$

Then, using Heisenberg's expression to describe the uncertainly associated with the position $\sigma_{X}$ and momentum $\sigma_{P}$ of a particle,

$$
\sigma_{X} \sigma_{P} \geq \frac{\hbar}{2}
$$

we may resolve physically significant values for $n_{L}, n_{M}$ and $n_{T}$. The uncertainty principle asserts a limit to the precision with which certain canonically conjugate pairs of particle properties can be known. Notably, the described uncertainty is with respect to the properties of position and momentum. We may consider, as such, describing each property as a variance of many possible certain measures. While the expression for variance is written to describe the certain properties of many particles, we modify this usage to describe the certain properties of many possible measurements whereby which measurement is applicable or even physically significant is uncertain.

$$
\sqrt{\frac{\sum_{i=1}^{N}\left(X_{i}-\bar{X}\right)^{2}}{N-1}} \sqrt{\frac{\sum_{i=1}^{N}\left(P_{i}-\bar{P}\right)^{2}}{N-1}}=\frac{\hbar}{2} .
$$

Such that all counts $N$ are reducible to a certain measure describing a single particle, we consider the case for $N=2$ random measures, although any value of $N$ will produce certain measures for all properties. Thus, the variances for position and momentum reduce such that there is a certain length $I$ corresponding to the variance in $X$ and a certain momentum $m v$ corresponding to the variance in $P$. As before, we write each variance in MQ nomenclature such that $l=n_{L r} l_{p}$ and $m v=m l / t=n_{M} m_{p}\left(n_{L} l_{p} / n_{T} t_{p}\right)$. Notably, the velocity change count $n_{L}$ is distinctly different than the position count $n_{L r}$, the latter which describes the distance between the observer and the particle.

$$
\sqrt{\left(n_{L r} l_{p}\right)^{2}} \sqrt{\left(n_{M} m_{p} \frac{n_{L} l_{p}}{n_{T} t_{p}}\right)^{2}}=\frac{\hbar}{2} .
$$


With these constraints, it follows that the position of a particle at the threshold $\hbar / 2$ is a certain distance $n_{L I} l_{p}$ from the observer. And the mass $n_{M} m_{p}$ and the velocity $n_{L} l_{p} / n_{T} t_{p}$ are products of their respective, certain, reference measures. Replacing the value $\hbar$ with the result from Eq. (5), then

$$
\begin{gathered}
\left(n_{L r} l_{p}\right)\left(n_{M} m_{p} \frac{n_{L} l_{p}}{n_{T} t_{p}}\right)=\frac{l_{p}^{2} m_{p}}{2 t_{p}}, \\
2 n_{L r} n_{M} n_{L}=n_{T} .
\end{gathered}
$$

Note also, the reference measures are no longer present (i.e. a geometric relation). This does not imply that the reference measures are physically significant or that the counts are integers. To resolve an understanding of what values are permitted, we will need new tools starting with a new description of $G$ that does not include Planck's reduced constant. Taking the Planck mass divided by the Planck length, then

$$
\begin{gathered}
\frac{m_{p}}{l_{p}}=\left(\frac{\hbar c}{G}\right)^{1 / 2}\left(\frac{c^{3}}{\hbar G}\right)^{1 / 2}=\frac{c^{2}}{G}, \\
G=\frac{c^{2} l_{p}}{m_{p}}=\frac{l_{p}^{2} l_{p}}{t_{p}^{2} m_{p}}=\frac{l_{p}^{3}}{t_{p}^{2} m_{p}}=\frac{l_{p}^{3} t_{p}}{t_{p}^{3} m_{p}}, \\
G=\left(\frac{l_{p}^{3}}{t_{p}^{3}}\right)\left(\frac{t_{p}}{m_{p}}\right) .
\end{gathered}
$$

With this, we consider two expressions at the $v=c$ bound also in terms of Planck Units, escape velocity and orbital motion. You will recognize the first as the Schwarzschild radius. When generalizing the values for any distance count $n_{L r}$ and any mass count $n_{M}$, then

$$
\begin{gathered}
v=\left(\frac{2 G M}{r}\right)^{1 / 2}, \\
c^{2}=\frac{2}{n_{L r} l_{p}}\left(\frac{l_{p}^{3}}{t_{p}^{3}} \frac{t_{p}}{m_{p}}\right) n_{M} m_{p}, \\
n_{L r}=2 n_{M} .
\end{gathered}
$$

Such that the orbital motion expression differs by a factor of two $v=(G M / r)^{1 / 2}$, the corresponding reduction follows the same path.

$$
n_{L r}=n_{M} \text {. }
$$

Recognition of the speed-of-light bound condition identifies a constraining relation between length and mass counts, specifically the length count $n_{L r}$ between the observer and a target with respect to the mass count $n_{M}$ corresponding to a gravitational mass. Use of the relation in Heisenberg's uncertainty expression constrains our understanding of the relation between these two phenomena. Starting with Heisenberg's reduced expression Equation (10) $2 n_{L r} n_{M} n_{L}=n_{T}$ and Equation (1) $n_{L}=n_{T}$, then 


$$
2 n_{L r} n_{M}=1 .
$$

At this time, we may apply the orbital motion expression to describe a particle's angular position with respect to a gravitational center or the escape velocity expression to describe a particle's linear position with respect to an observer along the path of that same vector. The latter is the required description of the relation with respect to the uncertainty principle. Thus, such that $n_{L r}=2 n_{M}$ from Equation (16), we make the substitution into Equation (18) and

$$
\begin{gathered}
2\left(2 n_{M}\right) n_{M}=1, \\
n_{M}^{2}=\frac{1}{4}, \\
n_{M}=\frac{1}{2} .
\end{gathered}
$$

The count value describes the lower count bound to the measure of mass with respect to an observer. This does not mean that phenomena may not have smaller masses, only that a mass less than $m_{f} / 2$ may not be measured with a greater precision. Notably, what is meant by direct measure is difficult and best understood by returning to the uncertainty principle for a precise definition. Returning to Equation (10) $2 n_{L r} n_{M} n_{L}=n_{T}$ such that $n_{M}=1 / 2$, moving $n_{L}$ to the right and reducing with Equation (1) $n_{L}=n_{T}$, then

$$
\begin{gathered}
2 n_{L r} \frac{1}{2} n_{L}=n_{T}, \\
n_{L r}=\frac{n_{T}}{n_{L}}=1,
\end{gathered}
$$

Finally, where both $n_{L}$ and $n_{L r}$ describe the phenomenon of length and Equation (1) $n_{L}=n_{T}$, then

$$
n_{L r}=n_{L}=n_{T}=1 .
$$

We conclude with this analysis that each of the counts is physically significant describing the boundary between certainty and uncertainty.

$O_{1}$ : There are physically significant fundamental units of measure: length, mass and time.

Notably, the mathematical approach taken makes no assumptions about the relations between the measures, the discreteness of measure or the physical significance of measure. Our ability to correlate a physically significant phenomenon with discrete counts of reference measures is entirely an outcome of our existing understanding of light, the uncertainty principle and escape velocity.

\subsection{Discreteness of Measure}

In the preceding section, we demonstrate that there is a lower count bound to measure. Consider now the physical significance of macroscopic measure (i.e. distance greater than the reference $l_{p}$ ). Imagine a stick $10.00 I_{p}$ in length and another $10.25 l_{p}$ in length. Can the difference be measured?

$$
10.25 l_{p}-10.00 l_{p}=0.25 l_{p} \text {. }
$$


No. A difference length is physically indistinguishable from any other length and with respect to the Heisenberg uncertainty principle, a distance less than the reference $l_{p}$ (i.e. $n_{L}=1$ ) cannot be measured. The demonstration constrains all macroscopic length measure to a whole-unit count of the reference measure.

While the above leaves no additional opportunity for non-discrete measure, consider one more approach, a difference greater than $l_{p}$ such that one stick is $10.00 I_{p}$ and the other is $15.25 I_{p}$.

$$
15.25 l_{p}-10.00 l_{p}=5.25 l_{p} .
$$

The difference measure is physically significant, but the argument that this measure is also non-discrete is valid only if different from a whole-unit count, that is five units of the reference. To test this prediction, we again compare the two targets,

$$
5.25 l_{p}-5.00 l_{p}=0.25 l_{p} .
$$

We find this case no different than the first. All measure is physically significant only where a whole-unit count of the reference. To summarize:

$\mathrm{O}_{2}:$ The fundamental measures are discrete and countable.

$\mathrm{O}_{3}$ : The fundamental measures each define a reference.

\subsection{Measurement Frames of Reference}

The prior observations establish a physically significant correlation between discrete measure and a reference. Consider now that the universe expands at the speed of light such that an observer within the universe has no access to information external to the universe. That is to say that there can be no external reference to the universe with respect to an observer within the universe. An experiment by Page and Wootters that supports a mechanism of "static" time was carried out demonstrating that time is a feature of the internal universe. This is demonstrated using the quantum entanglement of photons where it was shown that time is an emergent property deriving from quantum correlations [4].

In consideration of observations $O_{1}-O_{3}$ and the Page and Wootters result, we find support for the conjecture. Without a reference external to the universe, phenomena defined with respect to the universe are non-discrete. And as such, resolving a measurement difference between internal and external frames of reference will identify a physically significant result.

$O_{4}$ : Measure with respect to the observer is discrete.

$O_{5}$ : Measure with respect to the universe is non-discrete.

To test this prediction, consider first how we should describe the frame of an observer, a target and the universe in a single expression. The approach must allow for a perspective such that each of three measures is relatively known to the observer. And the approach must preserve the discrete reference measures.

As before, we propose that measure should be taken as a count of the reference. Just as one might use feet, meters, parsecs or light-years, we proceed with "units", units being defined as counts of the reference measures. Notably, we 
have not resolved the reference measures, but we will do so later. The exact values are not needed at this time.

Finally, we entertain the traditional approach to an unknown distance. As drawn in Figure 1 where our observer is at $A$ and the target is at $C$, the unknown distance count $\overline{B C}$ may be resolved with the reference count $\overline{A B}$, a discrete count of the reference count $\overline{A C}$ and an angle, each measure count resolved from the inertial frame of $A$. Thus

$$
\overline{B C}=\sqrt{\overline{A B}^{2}+\overline{A C}^{2}-2 \overline{A B A C} \cos \theta} .
$$

Although the approach can resolve each measure relative to the observer there are challenges. With the introduction of theta, we must address what a discrete length implies with respect to angular measure. That is to say, is an angular measure also discrete? We also find $\overline{B C}$ unconstrained where our goal is to narrow, not broaden, our understanding of the non-discrete segment.

As an alternative, consider now the case of an infinite grid of points all exactly one reference length apart with respect to their closest neighbors. At each point, an observer can use a laser pulse rangefinder and the time-of-flight principle to ascertain that they are at a distance of one $l_{p}$, hypothetically, from every other point, except those diagonal points which are calculated with the Pythagorean Theorem to be at a distance greater than the reference. In this example we also ascertain a right-angle design removing the need for an explicit measure of theta. And finally we change the location of the observer such that the observer, the target and the uncertain distance count all lie on a line representing the uncertain distance in the third frame of reference.

The example as drawn in Figure 2 demonstrates three distinct frames of

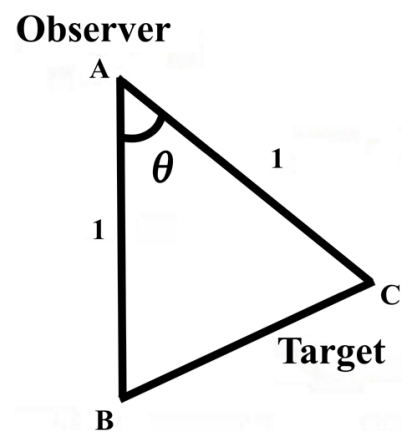

Figure 1. Count of distance measures along segment $\overline{B C}$.

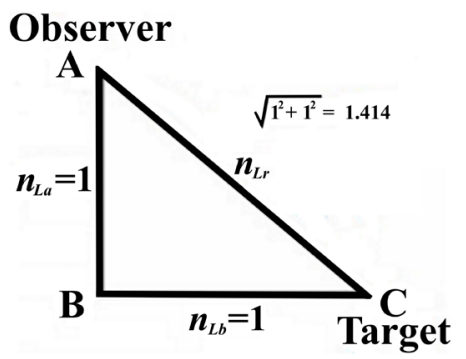

Figure 2. Count of distance measures along segment $\overline{A C}$. 
reference, the two discrete frames of $A$ and $C$ of which $A$ certifies the length of $\overline{A B}$ and $C$ certifies the length of $\overline{B C}$, and the third frame (the universe), of which both $A$ and $C$ and the unknown length $\overline{A C}$ are members. Using the Pythagorean Theorem, then $\overline{A C} \approx 1.414 l_{p}$. Such that only a discrete measure of $\overline{A C}$ is permitted, we then find the difference $1.414-1.000=0.414 l_{p}$ to describe a physically significant property of the universe. How this difference manifests between the distinct frames of $A$ and $C$ and the non-distinct frame $\overline{A C}$ of the universe is the subject of the section to follow.

\section{Results}

Using the principles discussed above we will now resolve a physical description of the difference between the discrete frame of the observer and the non-discrete frame of the universe. And with respect to both frames, we describe a common target.

\subsection{Distance}

To distinguish the approach from that of Planck, the fundamental units of measure derived will be identified with the subscript $f, l_{f}$ for length, $m_{f}$ for mass and $t_{f}$ for time. It is not necessary to resolve specific values yet. But, as the approach differs from that of Planck, it is important to distinguish between the measures.

Figure 3 describes the reference frames outlined in the prior section such that the long side $\mathrm{c}$ of a right-angle triangle may be resolved using the Pythagorean Theorem where side $a$ is always the reference count 1 and side $b$ is some known count of that reference.

Notice that a count of 1 on side $a$ is prerequisite to any definition of unknown factors. If an argument was presented that side a was arbitrary (i.e. $a=2$ ), we would find a description that "assumes" a reference count of two 1's not explicitly incorporated into the definition. This presents a factor representation of the framework that conceals the discrete count properties we are attempting to understand. Thus, side $\mathrm{a}=1$ is prerequisite for all considerations of side $\mathrm{b}$ in any understanding of the unknown distance on side $\mathrm{c}$.

$$
c=\left(1+n_{L b}^{2}\right)^{1 / 2} .
$$

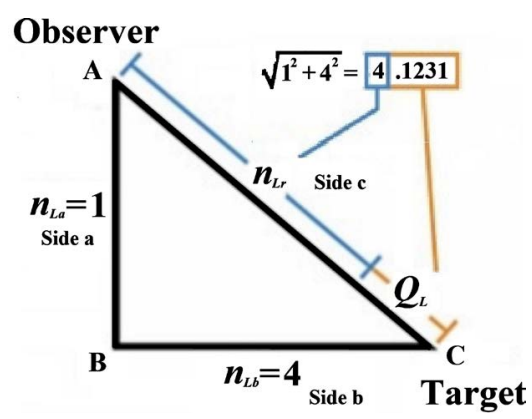

Figure 3. Count of distance measures between an observer and target. 
For the purposes of this approach, it is conjectured that any non-integer count relates to a change in distance and may be described by rounding up (repulsion) or down (attraction). The remainder lost to rounding will be denoted by $Q_{L}$. For all solutions, $Q_{L}$ is less than half and thus attractive as is evidenced by $Q_{L}$ 's largest value of 0.414 where sides $a$ and $b$ are both 1 . The model provides a count of distance measures that is closer by

$$
Q_{L}=\left(1+n_{L b}^{2}\right)^{1 / 2}-n_{L b}
$$

at every instant in time $t_{f}$ For example, if $n_{L b}=4$, then

$Q_{L} / n_{L b}=(\sqrt{17}-4) / 4=0.1231 / 4$. Because side $c$ always rounds down, we find that $n_{L r}$ always equals $n_{L b}$. Thus, we shall always refer to the 'observed measure count' as $n_{L r}$ Moreover, note that the reference measure against which all counts are measured is defined by $n_{L a}=1$. With this, we have composed an expression for gravity such that the loss of the remainder relative to the whole-unit count is $Q_{L} / n_{L r}$

Together $Q_{L}$ and $n_{L r}$ are conjectured to represent an important dimensionless ratio that describes gravity. We proceed with that hypothesis by presenting the ratio in meters per second squared $\left(\mathrm{ms}^{-2}\right)$, where we multiply by $l_{f}$ for meters and divide by $t_{f}^{2}$ together describing the distance loss at the maximum sampling rate of one sampling every $t_{f}$ seconds per second,

$$
\frac{Q_{L} l_{f}}{n_{L r} t_{f}^{2}} \text {. }
$$

Note also that the quantity is scaled and hence requires a scaling constant; we multiply by the speed of light $c$ and divide by a scaling constant $S$. Setting $r=$ $n_{L r} l_{f}$ and $c=I_{f} / t_{f}$ the expression reduces to

$$
\begin{aligned}
\frac{Q_{L} l_{f}}{n_{L r} t_{f}^{2}} \frac{c}{S}=\frac{Q_{L} c^{2}}{n_{L r} t_{f} S} & =\frac{Q_{L} l_{f} c^{2}}{n_{L r} l_{f} t_{f} S}=\frac{Q_{L} c^{3}}{r S} . \\
\frac{Q_{L} c^{3}}{r S} & \approx \frac{G}{r^{2}} .
\end{aligned}
$$

Notably, in Section III.D we will use Equation (13) $G=c^{3} t_{f} / m_{f}$ to demonstrate the relation between Equation (32) and $G$ directly through substitution. For now, we now have a description of gravitational curvature accurate with quantum precision for the entire physical domain.

We identify this presentation as the Informativity approach-a term that describes the application of measurement quantization to the description of phenomena. This understanding of gravity arises as a difference between the discrete measure of an inertial frame and the non-discrete measure with respect to the universe. Comparing the expression with Newton's expression $G / r^{2}$ we see a decrease in distance between the two curves (see Table 1) that quickly becomes immeasurable. The difference is a function of $Q_{L} n_{L r}$, a term that approaches $1 / 2$ with increasing distance as described in Appendix A.

But, before proceeding, let us take a closer look at the scalar constant $S$. 
Table 1. Informativity difference from $G / r^{2}$.

\begin{tabular}{ccccccc}
\hline & $50 l_{f}$ & $100 l_{f}$ & $300 l_{f}$ & $500 l_{f}$ & $1000 l_{f}$ & $2247 l_{f}$ \\
\hline Difference & $0.00100 \%$ & $0.00250 \%$ & $0.00028 \%$ & $0.00010 \%$ & $0.00003 \%$ & $0.00000 \%$ \\
\hline
\end{tabular}

\subsection{Scalar Constant $S$}

We may interpret $S$ as momentum; hence the units for the expressions above will match accordingly. We may also interpret $S$ as a radian measure. Let us consider both interpretations and the physical significance of each. To begin, we use Planck's expression for length to present one example of the relation between these two interpretations.

$$
l_{p} \frac{c^{3}}{G}=\frac{\hbar}{l_{p}}
$$

On the left, we have momentum. On the right, we have a description of Planck's reduced constant with respect to length. While Planck's constant $h$ carries the units joules seconds, Planck's reduced constant $\hbar=h / 2 \pi$ carries the units joules seconds per radian. The units for $\hbar / l_{p}$ are collectively recognized as angular momentum or more accurately the numerical value of momentum per radian.

By comparison, Equation (33) takes the same form. Solving first for $\mathcal{S}$, then substituting $r=n_{L r} l_{f}$ and considering macroscopic distances where

$$
\begin{aligned}
& \lim _{n_{L r} \rightarrow \infty} f\left(Q_{L} n_{L r}\right)=1 / 2 \quad \text { (Appendix A), then } \\
& S \approx \frac{Q_{L} r c^{3}}{G}=\frac{Q_{L} n_{L r} l_{f} c^{3}}{G}=\frac{1}{2} \frac{l_{f} c^{3}}{G}=\frac{\hbar}{2 l_{p}} .
\end{aligned}
$$

And as such, $S$ describes momentum just as much as it describes angular momentum, equal in magnitude but with respect to different dimensions. Is it always appropriate to consider one application? Do these different points of view vary with respect to different frames of reference?

Before beginning, note also that the term $Q_{L} n_{L r}$, referred to as the Informativity differential, plays a key role in describing how fractional values less than the theoretical limit describe a distorting effect in measurement. Consideration of the Informativity differential at a limit is a matter of convenience, but to maintain a precise expression, values for $Q_{L}$ and $n_{L r}$ should always be entered specifically to the phenomenon being observed. The values determined cover the entire physical domain from one $l_{f}$ to infinity.

Continuing our investigation of momentum, consider also this definition of mass. Multiply the left and right terms from the expression above by $t_{f}$ and reduce,

$$
m_{f}=t_{f} \frac{c^{3}}{G}=t_{f} \frac{2 S}{l_{f}}=\frac{2 S}{c} .
$$

Then the momentum is 


$$
\rho=m_{f} c=\left(\frac{2 S}{c}\right) c=2 S
$$

and may also be written as

$$
2 S=m_{f} c=\frac{m_{f} l_{f}}{t_{f}} .
$$

In passing, we have also confirmed our prior understanding of the minimum count value for $m_{f}$ as resolved in Equation (21). In reference to Equation (38) such that $m_{f} d_{f} / t_{f}$ describes the smallest units of length and time and such that $c=$ $n_{L} l_{f} / n_{T} t_{f}$ constrains $n_{L}=n_{T}=1$, then $n_{M}$ must equal one-half.

With this, we now investigate the angular properties of $S$. If we are to agree with the momentum interpretation as applied to Equation (35) $c^{3} / G=2 S / 1_{f}$ then the dimensional substitution $S=\hbar / 21_{f}$ from Planck's length expression Equation (2) $l_{f} c^{3} / G=\hbar / l_{f}$ must also be valid. As described in Figure 4 , then the arc length of a circle of radius $l_{f}$ and angle $S$ conforms to a radian measure.

$$
L=r \theta=r S=l_{f}\left(\frac{\hbar}{2 l_{f}}\right)=\frac{\hbar}{2} \text {. }
$$

To clarify, such that $r \theta$ meters radians describes the arc-length corresponding to a given radian measure and $\hbar / 2\left(\mathrm{~kg} \cdot \mathrm{m}^{2} \cdot \theta^{-1} \cdot \mathrm{s}^{-1}\right)$ describes a momentum per radian, then $S$ describes the magnitude of the angle in radians corresponding to the momentum of a quanta of energy. Writing down the corresponding units, cancelling out meters and moving $\theta$ to the left we find that

$$
\begin{gathered}
m \theta=\frac{\mathrm{kgm}^{2}}{\theta s}, \\
\theta^{2}=\frac{\mathrm{kgm}}{\mathrm{s}} .
\end{gathered}
$$

Notably, there is an argument with respect to the frame of the universe that the units for $r \theta$ are an arc-length per radian unit. In that case, $\theta$ cancels out and we recognize $\mathrm{kg} \cdot \mathrm{m} \cdot \mathrm{s}^{-1}$ as a unity expression equal to 1 , a dimensionless magnitude having no units.

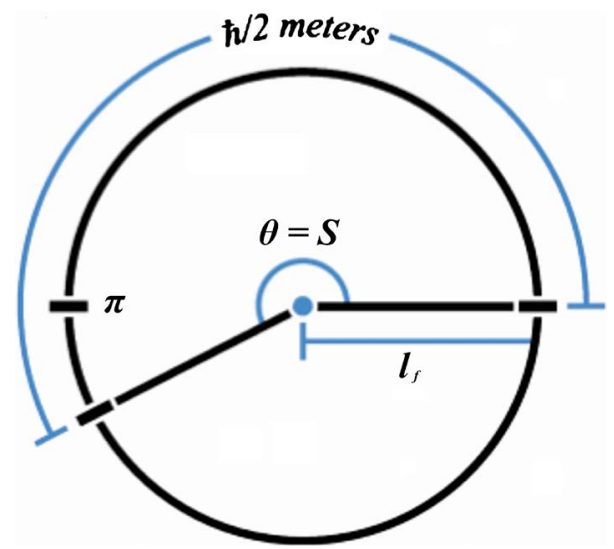

Figure 4. Arc length of a circle of radius $l_{f}$ and subtending angle $\theta=S$ radians. 
Alternatively, with respect to the inertial frame of the observer, the right-most term identifies the momentum interpretation from Equation (38) while the left-most term identifies an angular measure, neither being dominate. We recognize then,

$O_{6}:$ The scalar constant $S$ has units of $\mathrm{kg} \cdot \mathrm{m} \cdot \mathrm{s}^{-1}$, radians or no units at all depending on the frame of reference.

Naturally, the expression $l_{f} S=\hbar / 2$ is a numerical equality, so how can we test the dimensional validity of the angular approach to measure?

When working with the fundamental units of any expression dimensional validity may be resolved by replacing each dimension with the value of the corresponding fundamental measure. Such that there are $m_{f}$ kilograms, $I_{f}$ meters, $t_{f}$ seconds and $2 S$ radians where $2 S=\theta$ dimensionally then the conjecture that $2 S=\hbar / l_{f}=m_{f} l_{f} t_{f}$ is dimensionally valid is true only if

$$
\begin{gathered}
2 \theta=\frac{m_{f} l_{f}}{t_{f}}, \\
2 \times 3.26239=\frac{1.616199 \times 10^{-35} \times 2.17647 \times 10^{-8}}{5.39106 \times 10^{-44}}, \\
6.525=6.525 \text { radians. }
\end{gathered}
$$

By example only, using Planck Unit values from the 2014 CODATA [5], we confirm that the magnitude of $\theta$ is equivalent to that of momentum. Such that $S$ describes half the momentum of a fundamental unit of mass, then $2(1 / 2)=1^{2}$ is the only solution that can describe the dimensional equality of the fundamental units at unity. The test also confirms that $\theta$ must be a discrete value with respect to each of the fundamental measures. Unlike the measures of length, mass and time, this does not mean that $\theta$ is a measurement bound for all distance count $n_{L r}$ only that it is a lower bound with respect to $n_{L r}=1$.

Moreover, note that we are using the estimated 2014 Planck Unit values [5] which are sufficiently accurate for this demonstration. Later, we will resolve the fundamental unit values taking into account the effects of the Informativity differential. Those values are accurate to six significant digits and can be applied to any physical description to demonstrate dimensional homogeneity.

One might also inquire why this principle works. It is a mathematical outcome of the relation between each of the fundamental units of measure. When viewed from a more general perspective, the principle is no different than assigning each measure a random value. So long as the units are dimensionally equivalent and the expression balanced following assignment, then the expression will remain equal, regardless of the mathematical operations performed.

The question of initial dimensional equivalence is ensured by a second principle. Given a system of measurement, using the measured values for each fundamental unit validates the appropriateness and physical significance of each dimensional assignment. Any assignment that is inaccurate to a precision less than the remaining measures reduces the count of significant digits for all terms. 
Thus, to resolve the physical significance of a dimension at least all dimensions but one must be measured with a quantifiable physical significance. Then the significance of the remaining dimension must be equal or better than the most significant digit of the known physical measures. The above test tells us that the equivalence in the magnitude of radian measure and momentum is known to be physically significant to at least 4 significant digits and as previously noted, this can be shown to be significant to 6 digits when using the fundamental measure of each dimension.

One final note is of particular interest. In modern theory, we have associated Planck's reduced constant $\hbar$ with the momentum of a phenomenon per $2 \pi \approx$ 6.283 radians. In our analysis, we have resolved $\hbar$ with the momentum of a phenomenon per $2 S \approx 6.525$ radians. The $2 S$ interpretation corresponds to gravitational curvature, accurate with quantum precision throughout the entire physical domain. The two approaches agree on the value of $h$, but disagree on the best suited frame of reference for describing a "quantum of action". Forcing both results to a common frame produces evident discrepancies.

For instance, if we favor the modern interpretation (that a quantum of action strictly corresponds to $2 \pi$ radians), then we cannot agree that the momentum and angular measure expressions are mathematically equivalent. That is to say, such that the momentum $S=I_{f} \mathcal{B} / 2 G$ from Equation (35) and the angular measure $S=\hbar / 2 l_{f}$ from Equation (39) each share the scalar constant $S$, then their dimensional equality

$$
l_{f}=\left(\frac{\hbar G}{c^{3}}\right)^{1 / 2}
$$

must be wrong. But this is Planck's universally recognized formulation for length. And if this relation is askew, then all Planck Unit expressions are askew (not $h / 2 S$, rather $h / 2 \pi)$.

The issue is resolved only when we consider that two frames of reference are being considered. If one measures a 'quantum of action' equal to one full cycle $2 \pi$, then the proper expression is $\hbar=h / 2 \pi$. But, if one measures a "quantum of action" with respect to the frame of reference of the universe, then $\hbar_{f}=h / 2 S$ is of the proper magnitude. The difference varies as a function of the Informativity differential. To appropriately distinguish these two considerations, we distinguish the latter bound from Planck's reduced constant by appending the subscript $f\left(\right.$ i.e. $\left.\hbar_{f}\right)$, identifying the measure as Planck's fundamental constant. Specific physical examples where $\hbar_{f}$ applies will be discussed later in the paper.

\subsection{Measurement of $S$}

We will now consider an experiment where $S$ can be measured macroscopically with an optical parametric oscillator. That is, the properties of a pump beam may be controlled such that both the signal and idler demonstrate the unity state described above. And with each measure representing a count of one of the fundamental measures, $S$ is then a measure of the k vector angle of each of the split 
beams with respect to the atomic plane. Notably, when set up as described by Shwartz and Harris [6], solutions exist such that the signal is polarized in the scattering plane, the idler normal to the scattering plane and each a function of the current density of the pump.

Using polarization entangled X-Rays in pure Bell states, Shwartz and Harris take advantage of the intersections of the component curve (as a function of the square of the current density) to resolve the pump angle $\theta_{p}$ where the magnitudes of the components of each Bell state are equal. With this, solutions to the phase matching and current density equations are resolved to determine the sign of the components at the intersection. Then solving the phase matching equations for the signal and idler vectors with respect to the atomic plane, substituting the related electric fields, the current density is resolved entirely as a function of the pump.

We should note that the Shwartz and Harris calculations are dependent on electron charge and mass, collectively which provide a description of the electron density in the absence of the pumping beam. Naturally, one would need to correlate the gravitational expressions of Informativity with that of electromagnetism in order to carry out the same approach. This is possible with this description of the fine structure constant (FSC)

$$
\alpha=\frac{l_{f}}{a_{0}} \frac{m_{f}}{m_{e}}=\frac{1}{n_{M} n_{L}}=7.29710 \times 10^{-3}
$$

and our existing understanding of gravity

$$
\begin{gathered}
G=\frac{l_{f}}{m_{f} \varepsilon_{0} \mu_{0}}, \\
\frac{Q_{L} n_{L r} l_{f} c^{3}}{S}=\frac{l_{f}}{m_{f} \varepsilon_{0} \mu_{0}}, \\
2 S=m_{f} \varepsilon_{0} \mu_{0} c^{3} .
\end{gathered}
$$

The FSC is the inverse count product of the Bohr radius and the electron mass, resolved by dividing the Bohr radius by the fundamental length $n_{L}=a_{0} / l_{f}$ and the electron mass by the fundamental mass $n_{M}=m_{e} / m_{f}$ Likewise, the momentum of a fundamental unit of mass $2 S$ (i.e. the $k$ vector angle with respect to the polarization of the split beams and the scattering plane) is the product of that mass $m_{f}$ by the electromagnetic constants $\varepsilon_{0} u_{0}$ in three dimensions $c^{3}$ in the same manner as described by the gravitational constant $G=\left(t_{f} / m_{f}\right) c^{3}$.

Fortunately, Informativity offers an alternate solution to describe a maximally entangled Bell state with respect to a lattice vector $\boldsymbol{G}$. That is, we recognize as discussed by Shwartz and Harris that this occurs where one vector describes the polarization of the electric field in the scattering plane that the other describes the polarization orthogonal to the scattering plane. Thus, we only need to divide the magnitude of the pump vector coordinate components such that they are opposite in sign. Respectively, the $x$ and $y$ components are then the arccosine 
and arcsine of the lattice vector such that $\left(-n_{p x} \cos (\theta), n_{p y} \sin (\theta)\right)$. The pump, signal and idler vector magnitudes $n$ (a function of the pump frequency or the phase matching properties of the nonlinear optical crystal) are identified with the subscripts $p, s$ and $i$ followed by an $x$ or $y$ representing the coordinate axis.

$$
\begin{gathered}
\theta=\arccos \left(\frac{G_{x}}{n_{s x}+n_{i x}-n_{p x}}\right), \\
\theta=\arcsin \left(\frac{G_{y}}{n_{i y}+n_{i y}+n_{p y}}\right) .
\end{gathered}
$$

Placing these values in vector form and breaking out the component vectors, then

$$
\begin{gathered}
\left(\cos (\theta)\left(n_{s x}+n_{i x}-n_{p x}\right), \sin (\theta)\left(n_{i y}+n_{i y}+n_{p y}\right)\right)=\boldsymbol{G}, \\
\left(n_{s x} \cos (\theta)+n_{i x} \cos (\theta)-n_{p x} \cos (\theta), n_{i y} \sin (\theta)+n_{i y} \sin (\theta)+n_{p y} \sin (\theta)\right)=\boldsymbol{G}, \\
\left(n_{s x} \cos (\theta), n_{s y} \sin (\theta)\right)+\left(n_{i x} \cos (\theta), n_{i y} \sin (\theta)\right)-\left(n_{p x} \cos (\theta),-n_{p y} \sin (\theta)\right)=\boldsymbol{G}, \\
\left(n_{s x} \cos (\theta), n_{s y} \sin (\theta)\right)+\left(n_{i x} \cos (\theta), n_{i y} \sin (\theta)\right)-\left(n_{p x} \cos (\theta),-n_{p y} \sin (\theta)\right)=\boldsymbol{G} .
\end{gathered}
$$

Moving the pump coordinate to the right alongside of the lattice vector, taking the angular difference of the $y$-component to make the sine positive and matching that form in the $x$-component, then

$$
\begin{aligned}
& \left(n_{s x} \cos (\theta), n_{s y} \sin (\theta)\right)+\left(n_{i x} \cos (\theta), n_{i y} \sin (\theta)\right) \\
& =\left(n_{p x} \cos (2 \pi-\theta), n_{p y} \sin (2 \pi-\theta)\right)+\boldsymbol{G}
\end{aligned}
$$

We find that $\theta_{s}=\theta_{i}$ and that the pump angle is $\theta_{p}=2 \pi-\theta$. Moreover, such that the pump is split evenly, then the momentum of the beam is divided. As described in Equations ((37), (38)) we divide the momentum to match. Thus, when the angles of the $k$ vectors with respect to the atomic plane are equal to half the momentum of the entangled photons (i.e. S), then

$$
S=\frac{l_{f} c^{3}}{2 G}=\frac{1}{2}\left(m_{f} \frac{l_{f}}{t_{f}}\right) .
$$

Thus, the respective angles at maximal entanglement $\theta_{\text {Max }}$ associated with the signal and idler follow Equation (56) as described in line 2 of Table 2. An additional solution (line 1) may be resolved by subtracting each angle from $\pi$, (i.e. $\pi-$ $\left.\theta_{p}, \pi-\theta_{s}, \pi-\theta_{i}\right)$.

In Shwartz and Harris's 2011 paper, "Polarization Entangled Photons at

Table 2. Predicted radian measures of the $\mathrm{k}$ vectors of the pump, signal and idler for the maximally entangled state at the degenerate frequency of X-Rays.

\begin{tabular}{cccc}
\hline & $\theta_{p}$ & $\theta_{s}$ & $\theta_{i}$ \\
\hline$\pi-\theta_{\operatorname{Max}}$ & $\left(I_{f} \mathcal{c}^{3} / 2 G\right)-\pi(0.1208)$ & $\pi-\left(I_{f} \mathcal{c}^{2} / 2 G\right)(-0.1208)$ & $\pi-\left(I_{f} \mathcal{c}^{3} / 2 G\right)(-0.1208)$ \\
$\theta_{\operatorname{Max}}$ & $2 \pi-\left(I_{f} \mathcal{c}^{2} / 2 G\right)(3.02079)$ & $\left(I_{f} \mathcal{c}^{3} / G\right)(3.26239)$ & $\left(I_{f} \mathcal{c}^{3} / 2 G\right)(3.26239)$ \\
\hline
\end{tabular}


$X$-Ray Energies" [6], they resolve a total of five cases representing two Bell states that can generate entangled photons. Using their nomenclature, the states are defined such that $|H\rangle$ is the polarization of the electric field of the X-ray in the scattering plane and $|V\rangle$ is the polarization orthogonal to the scattering plane which contains the incident $k$ vector and the lattice $k$ vector $\boldsymbol{G}$.

The Shwartz and Harris measure precisely match the Informativity calculations (Table 3) confirming the predictions described by Informativity to six significant digits, which is the extent of precision allowed by $G$. Moreover, the error in angular measure for the Shwartz and Harris results is estimated to be less than 2 micro-radians.

Of particular interest are the component terms that define the scalar constant $S=I_{f} \mathcal{C}^{3} / 2 G$ : the Planck length $l_{p}$, the speed of light $c$ and the gravitational constant $G$. Using the 2014 CODATA [5] values for guidance, then

$$
S=\frac{l_{f} c^{3}}{2 G}=\frac{1.616199 \times 10^{-35} \times 299792458^{3}}{2 \times 6.67408 \times 10^{-11}}=3.26239 \text { radians . }
$$

The role of fundamental measures to this point is a mathematical construct, a proposed interpretation of the existing argument. The measures exist only in their expression until formally resolved in the next section. Whereas CODATA estimates may be used to guide our understanding of $S$, up to this point no theoretical values are assumed. Our confidence in correlating $S$ to $\theta_{s i}$ rests in the correctness of the two interpretations of $S$, their correlation which accounts for Planck's length expression, the resulting measurement predictions and the collaborating results presented by Shwartz and Harris.

\subsection{Discrete Measures}

In modern theory, we quantify the relation between length and time with respect to the speed of light $c=1 / t$. But mass stands alone, without a specific phenomenon correlating mass to length or time. To resolve all three of the fundamental measures will require a correlation. Starting with the geometric approach presented in Section III. A $Q_{L} c^{3} / r \theta_{s i}$ and its correlation to the gravitational constant $G$ Equation (33), then removing the Informativity differential $\lim _{n_{L r} \rightarrow \infty} f\left(Q_{L} n_{L r}\right)=1 / 2$ (Appendix A) - a physically significant equivalent for any macroscopic distance-it follows that

$$
G \approx r^{2} \frac{Q_{L} c^{3}}{r \theta_{s i}}=n_{L r} l_{f} \frac{Q_{L} c^{3}}{\theta_{s i}}=\left(Q_{L} n_{L r}\right) \frac{l_{f} c^{3}}{\theta_{s i}}=\frac{l_{f} c^{3}}{2 \theta_{s i}} .
$$

And, such that $G=c^{3} t_{f} / m_{f}$ from Equation (13), then

Table 3. Angle setting in radians of the $\mathrm{k}$ vectors of the pump, signal and idler for maximally entangled states at the degenerate frequency (Ref. [6]).

\begin{tabular}{cccc}
\hline Bell's State & $\theta_{p}$ & $\theta_{s}$ & $\theta_{i}$ \\
\hline$\left(\left|H_{s}, V_{i}\right\rangle+\left|V_{s}, H_{i}\right\rangle\right) / \sqrt{2}$ & 0.1208 & -0.1208 & -0.1208 \\
& 3.02079 & 3.26239 & 3.26239 \\
\hline
\end{tabular}




$$
\begin{gathered}
\frac{t_{f} c^{3}}{m_{f}} \approx \frac{l_{f} c^{3}}{2 \theta_{s i}}, \\
l_{f} m_{f} \approx 2 \theta_{s i} t_{f} .
\end{gathered}
$$

We call this the fundamental expression.

Although a macroscopic expression for fundamental length may be resolved directly from Equation (33), we will start with the initial geometric formulation finalized in Equation (32) and our understanding of $G=c^{3} t_{f} / m_{f}$ Equation (13) to directly correlate gravity through substitution. This can help significantly reduce the number of considerations to this point. Breaking down the expression, applying the macroscopic case for the Informativity differential $\lim _{n_{L r} \rightarrow \infty} f\left(Q_{L} n_{L r}\right)=1 / 2$, we resolve fundamental length.

$$
\begin{gathered}
\frac{Q_{L} c^{3}}{r \theta_{s i}}=\frac{Q_{L} c^{3}}{n_{L r} l_{f} \theta_{s i}}=\frac{Q_{L}}{n_{L r} l_{f} \theta_{s i}} \frac{c^{3} t_{f}}{m_{f}} \frac{m_{f}}{t_{f}}, \\
\frac{Q_{L} c^{3}}{r \theta_{s i}}=\frac{Q_{L}}{n_{L r} l_{f} \theta_{s i}} G \frac{m_{f}}{t_{f}}, \\
l_{f}=\frac{r \theta_{s i}}{Q_{L} c^{3}} \frac{Q_{L}}{n_{L r} \theta_{s i}} G \frac{m_{f}}{t_{f}}, \\
l_{f}=\frac{n_{L r} l_{f} \theta_{s i}}{Q_{L} c^{3}} \frac{Q_{L}}{n_{L r} \theta_{s i}} G \frac{m_{f}}{t_{f}}=G \frac{m_{f}}{c^{2}}, \\
l_{f}=G \frac{1}{c^{2}} \frac{2 \theta_{s i} t_{f}}{l_{f}}=\frac{2 G \theta_{s i}}{c^{3}} .
\end{gathered}
$$

Then, for all macroscopic distances, the fundamental units are

$$
\begin{gathered}
l_{f}=\frac{2 G \theta_{s i}}{c^{3}}=\frac{2 \times 6.67408 \times 10^{-11} \times 3.26239}{299792458^{3}}=1.61620 \times 10^{-35} \mathrm{~m}, \\
t_{f}=\frac{l_{f}}{c}=\frac{2 G \theta_{s i}}{c^{4}}=\frac{2 \times 6.67408 \times 10^{-11} \times 3.26239}{299792458^{4}}=5.39106 \times 10^{-44} \mathrm{~s}, \\
m_{f}=\frac{2 \theta_{s i}}{c}=t_{f} \frac{c^{3}}{G}=\frac{2 \times 3.26239}{299792458}=2.17643 \times 10^{-8} \mathrm{~kg} .
\end{gathered}
$$

Notably, when we say macroscopic, we mean any distance greater than $2247 l_{f}$ as described in Table 1 . For any distance greater than this, the geometric skew due to the Informativity differential is a value less than 0.5 of the sixth digit of physical significance. To resolve a form of this expression accurate with greater precision, the fundamental length may be written as

$$
l_{f}=\frac{G \theta_{s i}}{Q_{L} n_{L r} c^{3}}=\left(c^{3} \frac{t_{f}}{m_{f}}\right) \frac{\theta_{s i}}{Q_{L} n_{L r} c^{3}}=\frac{\theta_{s i} t_{f}}{Q_{L} n_{L r} m_{f}} .
$$

The fundamental expression in "expanded form"-the term used when applying this effect-is written as

$$
Q_{L} n_{L r} l_{f} m_{f}=\theta_{s i} t_{f}
$$


As well and in prior publications, we often use the equal sign, as opposed to the approximately equal sign when working with the macroscopic form of these expressions. This is an acceptable practice so long as one bears in mind that the more accurate representation requires consideration of the Informativity differential.

\subsection{Physically Significant Discrepancies with $\hbar$}

The conflicts that Planck encountered arose from the missing experimental connection to the effects of distance measure on $G$ and $\hbar$. Whereas the measure of $\hbar$ is a property of quantum interactions, the measure of $G$ is resolved as a property of macroscopic phenomena. Mixing the two distance sensitive values in a single expression as Planck defines his units leads to incorrect physical predictions. One example includes the calculation of $G$. To resolve the issue, we solve a distance sensitive value for $\hbar$ ( $\hbar \approx 2 \theta_{s i} I_{f}$ Equation (39)) such that $\lim _{n_{L r} \rightarrow \infty} f\left(Q_{L} n_{L r}\right)=1 / 2$, then for any macroscopic set of measures (i.e. $G$ )

$$
\hbar_{f}=\frac{\theta_{s i} l_{f}}{Q_{L} n_{L r}}=2 \theta_{s i} l_{f}=1.05454 \times 10^{-34} \mathrm{~J} \cdot \mathrm{s} .
$$

Importantly, the value is resolved solely as a function of $\theta_{s i}$ and $l_{f}$ as derived from the initial geometric argument. This significantly reduces dependencies placing focus on the physical measurement of $\theta_{s i}$ and the measure of light $c=1 / t$ which may now be approached as a count property of the Heisenberg uncertainty principle. The most notable discrepancies-length and time-with respect to the 2014 CODATA (Table 4) in comparison to Planck's Unit expressions Equations ((2), (3)) are now reduced to the last significant digit.

Notably, there is a very small rounding effect taking place in length that is then amplified in the mass. Half the difference between the $\hbar_{f}$ calculation and the CODATA length value is $0.0000006 \times 10^{-35} \mathrm{~m}$. Had this difference been just below $0.0000005 \times 10^{-35} \mathrm{~m}$ the rounding would have matched the CODATA precisely. But, in neither case is the seventh digit physically significant and as such should not be considered. Given that $m_{f}=2 \theta_{s i} t_{f} I_{f}$ the difference in the mass from the CODATA is then an amplification of the rounding in the length, precisely.

With a distance sensitive measure for Planck's reduced constant adjusted for the Informativity differential, we may now describe in Table 5 the predicted radian measures presented in Table 2 as a function of $\hbar_{f}$

Table 4. Planck's expressions calculated with quantum $\hbar$ and macroscopic $\hbar_{f}$ values for Planck's constant.

\begin{tabular}{ccccc}
\hline Informativity Differential & $\theta$ (radians) & Length $(\mathrm{m})$ & Mass $(\mathrm{kg})$ & Time $(\mathrm{s})$ \\
\hline Planck's Reduced Constant $\hbar$ & 3.26250 & $1.616228 \times 10^{-35}$ & $2.17647 \times 10^{-8}$ & $5.39116 \times 10^{-44}$ \\
Planck's Fundamental Constant $\hbar_{f}$ & 3.26239 & $1.616200 \times 10^{-35}$ & $2.17643 \times 10^{-8}$ & $5.39106 \times 10^{-44}$ \\
2014 CODATA Estimates [5] & & $1.616199 \times 10^{-35}$ & $2.17647 \times 10^{-8}$ & $5.39106 \times 10^{-44}$ \\
\hline
\end{tabular}


Table 5. Predicted radian measures of the $\mathrm{k}$ vectors of the pump, signal and idler for the maximally entangled state at the degenerate frequency of X-Rays using Planck's fundamental constant $\hbar_{f}$

\begin{tabular}{cccc}
\hline & $\theta_{p}$ & $\theta_{s}$ & $\theta_{i}$ \\
\hline$\pi-\theta_{\operatorname{Max}}$ & $\left(\hbar_{f} / 2 I_{f}\right)-\pi(0.1208)$ & $\pi-\left(\hbar_{f} / 2 I_{f}\right)(-0.1208)$ & $\pi-\left(\hbar_{f} / 2 I_{f}\right)(-0.1208)$ \\
$\theta_{\operatorname{Max}}$ & $2 \pi-\left(\hbar_{f} / 2 I_{f}\right)(3.02079)$ & $\left(\hbar_{f} / 2 I_{f}\right)(3.26239)$ & $\left(\hbar_{f} / 2 I_{f}\right)(3.26239)$ \\
\hline
\end{tabular}

For a more in-depth understanding of the effects of the Informativity differential on $G$ and $\hbar$, we present the following relation which shows their correlation. Starting with the fundamental expression and Equation $(39) \hbar \approx 2 \theta_{s i} I_{\text {f }}$ then

$$
\begin{gathered}
2 \theta_{s i} t_{f} \approx l_{f} m_{f}, \\
4 \theta_{s i}^{2} t_{f} \approx\left(2 \theta_{s i} l_{f}\right) m_{f}, \\
4 \theta_{s i}^{2} t_{f} \approx \hbar m_{f}, \\
4\left(c^{3} \frac{t_{f}}{m_{f}}\right) \theta_{s i}^{2} \approx \hbar c^{3}, \\
4 G \theta_{s i}^{2} \approx \hbar c^{3} .
\end{gathered}
$$

Notably, moving terms to the left to match the expression for fundamental length, we find Planck's terms appear on the right matching the expression for Planck's length. Where Planck's expressions have no specific correlation to physical measure, the Informativity expression is correlated to gravitational curvature to the sixth significant digit as demonstrated with the combination of Equation (13) $G=c^{3} t_{f} / m_{f}$ with Equation (32) $Q_{L r} c^{3} / r \theta_{s i}$ which demonstrates that $G=c^{3} 1 / 2 \theta_{s i}$ Equations (62)-(66). Thus,

$$
\begin{gathered}
\frac{4 G \theta_{s i}^{2}}{c^{3}} \approx \hbar, \\
\frac{4 G^{2} \theta_{s i}^{2}}{c^{6}} \approx \frac{\hbar G}{c^{3}}, \\
\frac{2 G \theta_{s i}}{c^{3}} \approx\left(\frac{\hbar G}{c^{3}}\right)^{1 / 2} .
\end{gathered}
$$

The challenge Planck encountered was a quantum variation (an observational skew) in $G$ and $\hbar$ equal in magnitude that are related as described in Equation (77). Without expressions and experimental data describing the variation, he lacked a foundation with which to resolve a distance sensitive understanding of $\hbar_{f}$ macroscopically. Where $\left(\hbar G / c^{3}\right)^{1 / 2}=1.61623 \times 10^{-35} \mathrm{~m}$, using the Planck fundamental constant $\hbar_{f}$ the above expression is now mathematically equivalent

$$
\begin{gathered}
\frac{2 \times 6.67408 \times 10^{-11} \times 3.26239}{299792458^{3}} \approx\left(\frac{1.05454 \times 10^{-34} \times 6.67408 \times 10^{-11}}{299792458^{3}}\right)^{1 / 2}, \\
1.61620 \times 10^{-35} \approx 1.61620 \times 10^{-35} .
\end{gathered}
$$

Moreover, the physical conflict with experimental result is resolved. As origi- 
nally described in Equation (39) $\theta_{s i}=\hbar / 2 l_{f}=3.26250$ radians, accounting for the Informativity differential solves the discrepancy with respect to the Shwartz and Harris results.

$$
\theta_{s i}=\frac{\hbar_{f}}{2 l_{f}}=\frac{1.05454 \times 10^{-34}}{2 \times 1.616199 \times 10^{-35}}=3.26239 \text { radians . }
$$

The dimensional homogeneity problem is solved as expressed in Equations (42)-(44). Presenting each of the fundamental measures as described by the fundamental expression, $2 \theta_{s i}=l_{f} m_{f} / t_{f}$ we now find the expression mathematically equal and in correspondence with the 2014 CODATA measurements [5],

$$
\begin{gathered}
2 \times 1 \times 3.26239=\frac{1.616200 \times 10^{-35} \times 2.17643 \times 10^{-8}}{5.39106 \times 10^{-44}}, \\
6.52548=6.52548 \text { radians } .
\end{gathered}
$$

The same follows from $G$ when resolved as a measure of the quantum value $\hbar$. Using the 2014 CODATA for Planck's length $l_{p}$ and $\hbar$ in Planck's expression for length $G=c^{3} l_{p}^{2} / \hbar=6.67385 \times 10^{-11}$ in comparison with the distance sensitive measure of $\hbar_{f}$ then

$$
G=\frac{c^{3} l_{f}^{2}}{\hbar_{f}}=\frac{299792458^{3} \times\left(1.616200 \times 10^{-35}\right)^{2}}{1.05454 \times 10^{-34}}=6.67408 \times 10^{-11} \mathrm{~m}^{3} \cdot \mathrm{kg}^{-1} \cdot \mathrm{s}^{-2} .
$$

The Informativity approach conforms to the measurement data (i.e. $6.67408 \times$ $10^{-11} \mathrm{~m}^{3} \cdot \mathrm{kg}^{-1} \cdot \mathrm{s}^{-2}$ ) [5] while use of the Planck reduced constant $\hbar$ demonstrates a physically significant discrepancy of $0.00023 \times 10^{-11} \mathrm{~m}^{3} \cdot \mathrm{kg}^{-1} \cdot \mathrm{s}^{-2}$.

Moreover, the Informativity approach allows calculation of $G$ entirely as a function of $l_{f}$ the one-to-one relation of $l_{f}$ to $t_{f}\left(\right.$ i.e. $\left.l_{f}=t_{f} \mathcal{c}\right)$ and $\theta_{s i}$.

$$
\begin{gathered}
Q_{L}=n_{L c}-n_{L b}=\sqrt{1+n_{L b}^{2}}-n_{L b}, \\
Q_{L}=\sqrt{1+\left(6.18735 \times 10^{34}\right)^{2}}-6.18735 \times 10^{34}=8.08100 \times 10^{-36}, \\
\frac{Q_{L} c^{3}}{r S}=\frac{8.08100 \times 10^{-36} \times 299792458^{3}}{1 \times 3.26239}=6.67408 \times 10^{-11} \mathrm{~m} / \mathrm{kg} \cdot \mathrm{s}^{2} .
\end{gathered}
$$

And finally, returning to the long-running discrepancy between the Planck expression for the FSC $\alpha=l_{f} m_{f} / a_{0} m_{e}=7.29710 \times 10^{-3}$ and its 2014 CODATA measure $7.29735 \times 10^{-3}$, we incorporate the Informativity differential at a distance respective of the measure of Planck's reduced constant (Appendix B) [[1], see Equation (34)].

$$
r_{L f}=\theta_{s i} l_{f} \sqrt{\frac{1}{\hbar\left(\hbar-2 \theta_{s i} l_{f}\right)}}=84.85536 .
$$

The value identifies the equivalent count of $l_{f}$ that corresponds to the birth of a photon. Then, expanding the Planck expression for the FSC using the fundamental expression $l_{f} m_{f}=\theta_{s i} t_{f} / Q_{L} n_{L I}$, the distance sensitive value is resolved. 


$$
\alpha=\frac{l_{f} m_{f}}{a_{0} m_{e}}=\frac{1}{a_{0} m_{e}} \frac{\theta_{s i} t_{f}}{Q_{L} n_{L r}}=7.29735 \times 10^{-3} .
$$

The result is a precise match to the 2014 CODATA value.

The strict mathematical constraints of this relation draw immediate attention to the many physically significant discrepancies that arise with the introduction of Planck's reduced constant unadjusted for the measurement skewing effects described by the Informativity differential.

\subsection{Direct Measurement of the Informativity Differential}

The Informativity differential describes a skewing effect in our understanding of distance. The effect is very small, but a measure of this effect is just now becoming possible with new data expected from the GAIA mission [7]. Measured with respect to Einstein's calculation of the effects of relativity on light passing through a gravitational field, $\gamma$ is the difference from that calculation. The most cited and currently most accurate measure of $\gamma$ is $(21 \pm 23) \times 10^{-6}$ [8] with respect to measurements made of transmissions from the Cassini spacecraft. With Informativity we may calculate the results of $\gamma$ which will show a value equal to the Informativity differential.

We use the expressions for the deflection of light grazing our Sun to resolve $\gamma$ as a difference from Einstein's expressions. With $\theta$ the angle of deflection, $r$ and $M_{\odot}$ the radius and mass of our sun, $G$ the gravitational constant, and c the speed of light, then

$$
\theta=\frac{4 G M_{\square}}{r c^{2}}=\frac{G}{r^{2}} \frac{4 r M_{\square}}{c^{2}}=8.5 \times 10^{-6} \text { radians . }
$$

Taking the difference between $G / r^{2}$ and the Informativity expression $Q_{L f} c^{3} / r \theta_{s i}$ we may resolve the radian difference between GR and Informativity,

$$
\Delta \theta=\left(\frac{G}{r^{2}}-\frac{Q_{L f} c^{3}}{r \theta_{s i}}\right) \frac{4 r M}{c^{2}}=\frac{4 M\left(G \theta_{s i}-Q_{L f} r c^{3}\right)}{r c^{2} \theta_{s i}}=6.6 \times 10^{-12} \text { radians . }
$$

The effect resolves to six orders in magnitude less than the effects of GR or $\gamma=0.78 \times 10^{-6}$. While the GAIA mission will certainly offer enticing data with respect to our understanding of $\gamma$, a definitive result will need to await a mission with one order increase in precision.

\section{Discussion}

Organizing the fundamental expression such that $c=I_{f} / t_{f}=2 \theta_{s i} / m_{f}$ allows us to state that any change in the count of discrete units of length must equal the same in a count of discrete units of time for any description of light in a system defined relatively. The relative nature of discrete behavior is crucial because as noted earlier, measure is a function of references in the local inertial frame with respect to a target. But, what if the observed phenomenon is a combination of the effects of relative measure on top of characteristics a function of and with respect to the universe? 
The question invites again the required understanding as resolved herein, that is, our ability to resolve certain properties of the universe, such as its diameter, mass and age as observed from outside with respect to an external reference. In that the universe expands at the speed of light there is no possibility for a reference external to the universe to play a part in the observer's understanding of a phenomenon. Moreover, we also recognize that discreteness is a property of references. Thus, while matter within the universe may relatively take on discrete behavior, relative to the universe behavior must be non-discrete. The two reference frames express themselves, for one, as a lost difference in distance which we observe as gravity.

Discreteness is not only a function of references with respect to the inertial frame. It is a function of the information available. While it may be argued that all observers have a precise understanding of the three measures, some information may not be available at a given point in space-time. And as such, the missing information will result in a non-discrete, non-deterministic behavior, for example, for any behavior that takes on a measure less than the whole-unit values in Heisenberg's reduced particle expression $2 n_{M} n_{L I} n_{L} \geq n_{T}$.

The significance of discrete measure identifies a new behavior essential to our understanding of physical interactions. Measure is bounded to whole-unit counts of the reference measures and those bounds constrain the physical regime eliminating the conditions that lead to singularities. Collectively $l_{f} / t_{f} m_{f} / t_{f}$ and $m_{f} / l_{f}$ identify those bounds.

\section{Conflicts of Interest}

The authors declare no conflicts of interest regarding the publication of this paper.

\section{References}

[1] Geiger, J.A. (2018) Measurement Quantization Unites Classical and Quantum Physics. Journal of High Energy Physics Gravitation and Cosmology, 4, 262-311. https://doi.org/10.4236/jhepgc.2018.42019.

[2] Geiger, J.A. (2018) Measurement Quantization Unifies Relativistic Effects, Describes Inflation/Expansion Transition, Matches CMB Data. Journal of High Energy Physics Gravitation and Cosmology, 4, 655-694. https://doi.org/10.4236/jhepgc.2018.44038.

[3] Geiger, J.A. (2019) Measurement Quantization Describes Galactic Rotational Velocities, Obviates Dark Matter Conjecture. Journal of High Energy Physics Gravitation and Cosmology, 5, 473-506. https://doi.org/10.4236/jhepgc.2019.52028

[4] Moreva, E., et al. (2014) Time from Quantum Entanglement: An Experimental Illustration. Physical Review A, 89, Article ID: 052122. arXiv: 1310.4691. https://doi.org/10.1103/PhysRevA.89.052122

[5] Mohr, P., Taylor, B. and Newell, D. (2015) CODATA Recommended Values of the Fundamental Physical Constants: 2014. arXiv: 1507.07956v1.

https://doi.org/10.6028/NIST.SP.961r2015

[6] Shwartz, S. and Harris, S.E. (2011) Polarization Entangled Photons at X-Ray Ener- 
gies. Physical Review Letters, 106, Article ID: 080501. arXiv: 1012.3499. https://doi.org/10.1364/NLO.2011.NWC3

[7] Will, C.M. (2014) The Confrontation between General Relativity and Experiment. Living Reviews in Relativity, 17, 4. https://doi.org/10.12942/lrr-2014-4

[8] Bertotti, B., Iess, L. and Tortora, P. (2003) A Test of General Relativity Using Radio Links with the Cassini Spacecraft. Nature, 425, 374-376.

https://doi.org/10.1038/nature01997 


\section{Appendices}

\section{Appendix A: Numerical Limits to $Q_{L} n_{L r}$}

Throughout the paper, we find the term $Q_{L} n_{L r}$ repeatedly. This term is referred to as the informativity differential in recognizing the central role it plays in describing how fractional values less than the theoretical limit reflect a distortion effect in distance measurement. Knowing the limits to $Q_{L} n_{L r}$ is also essential in resolving the fundamental measures.

The product of $Q_{L} n_{L r}$ is Equation (30) multiplied by $b$.

$$
Q_{L} n_{L r}=\left(\sqrt{1+n_{L r}^{2}}-n_{L r}\right) n_{L r}
$$

Note, what is measured always equals a whole-unit count of a fundamental measure, and with $a=1$ we find that $b=n_{L r}$ for all values. This is easily verified in that the highest value for $Q_{L}$ is obtained for $b=1$ where $\left(1+1^{2}\right)^{0.5}-1=0.414$ and the 'observed' distance of $c$ presented as a count $n_{L r}$ is always rounded down to the highest integer value equal to the count $b$ with $Q_{L}=0.414$ at its highest and quickly approaching 0 with increasing $b$. Therefore,

$$
Q_{L} n_{L r}=\left(\sqrt{1+n_{L r}^{2}}-n_{L r}\right) n_{L r}
$$

The lower limit where $n_{L r}=1$ is easily produced, $\lim _{r=1} f\left(Q_{L} n_{L r}\right)=\sqrt{2}-1$. Conversely, if we divide by $n_{L r}$, then add $n_{L r}$, square, subtract $n_{L r}^{2}$, and divide by 2 , we find that

$$
\frac{Q_{L}^{2}}{2}+Q_{L} n_{L r}=\frac{1}{2}
$$

$Q_{L}$ decreases with increasing $n_{L r}$ until the left term drops out. Distance does not need to be significant to reduce the Informativity differential to 0.5. At just $10^{4} I_{f} Q_{L} n_{L r}$ rounds to 0.5 to nine significant digits.

\section{Appendix B: Effective Count of $l_{f}$ in the Measure of $\hbar$}

The measure of Planck's constant requires a physical interaction at a specific relative distance. That distance may be resolved as a count of $l_{f}$ using Equation (30) where $n_{L b}$ equals to $n_{L r}$ and Equation (35) where we have substituted $\hbar / l_{f}^{2}$ from Planck's relation in Equation (2). We have

$$
\begin{gathered}
Q_{L f}=\left(1+r_{L f}^{2}\right)^{1 / 2}-r_{L f}, \\
\theta_{s i}=\left(\frac{c^{3}}{G}\right) Q_{L f} r_{L f} l_{f}=\left(\frac{\hbar}{l_{f}^{2}}\right) Q_{L f} r_{L f} l_{f}, \\
r_{L f}=\frac{\theta_{s i} l_{f}}{\hbar Q_{L f}}=\frac{\theta_{s i} l_{f}}{\hbar\left(\left(1+r_{L f}^{2}\right)^{1 / 2}-r_{L f}\right)}, \\
\left(r_{L f}^{2}+r_{L f}^{4}\right)^{1 / 2}-r_{L f}^{2}=\frac{\theta_{s i} l_{f}}{\hbar},
\end{gathered}
$$




$$
\begin{gathered}
r_{L f}^{2}+r_{L f}^{4}=\left(\frac{\theta_{s i} l_{f}}{\hbar}+r_{L f}^{2}\right)^{2}=\frac{\theta_{s i}^{2} l_{f}^{2}}{\hbar^{2}}+\frac{2 \theta_{s i} l_{f} r_{L f}^{2}}{\hbar}+r_{L f}^{4}, \\
r_{L f}^{2}\left(1-\frac{2 \theta_{s i} l_{f}}{\hbar}\right)=\frac{\theta_{s i}^{2} l_{f}^{2}}{\hbar^{2}}, \\
r_{L f}=\sqrt{\frac{\theta_{s i}^{2} l_{f}^{2}}{\hbar^{2}\left(1-\frac{2 \theta_{s i} l_{f}}{\hbar}\right)}}=\theta_{s i} l_{f} \sqrt{\frac{1}{\hbar\left(\hbar-2 \theta_{s i} l_{f}\right)}} .
\end{gathered}
$$

